\title{
GAIT AND MUSCLE STRENGTH CHARACTERISTICS IN TOTAL KNEE ARTHROPLASTY PATIENTS WITH PATELLOFEMORAL PAIN SYNDROME BEFORE AND SIX MONTHS AFTER SURGERY
}

\author{
H. Gapeyeva ${ }^{1,2}$, J. Ereline ${ }^{1,2}$, T. Haviko ${ }^{3}$, H. Aibast ${ }^{1,2}$, \\ M. Pääsuke ${ }^{1,2}$ \\ ${ }^{1}$ Institute of Exercise Biology and Physiotherapy, \\ University of Tartu, Tartu, Estonia \\ ${ }^{2}$ Centre of Behavioural and Health Sciences, \\ University of Tartu, Tartu, Estonia \\ ${ }^{3}$ Department of Traumatology and Orthopaedics, \\ University of Tartu, Tartu, Estonia
}

\begin{abstract}
The aim of the present study was to investigate the gait and muscle strength characteristics in total knee arthroplasty (TKA) patients with patellofemoral pain syndrome (PFPS) before and six months after surgery. Eight patients (4 men and 4 women) aged 58-77 years with PFPS following unilateral TKA who had primary degenerative knee OA participated in the study before and six months after reoperation. In patients was registered the active range of motion (AROM) of knee extension and flexion, hip abduction and adduction. Isometric maximal voluntary contraction (IMVC) force of knee flexors, extensors, abductors and adductors was measured and gait kinematic characteristics and kinetic characteristics of knee joint were recorded. Knee flexion AROM in the involved leg was significantly lower $(p<0.05)$ as compared to the uninvolved leg pre- and post-surgery. The patients had greater $(p<0.05)$ hip abduction AROM and significant increase $(31 \%, p<0.05)$ of IMVC force of the involved leg's hip abductors postoperatively as compared before surgery. Six months after surgery
\end{abstract}


a significant $(p<0.05)$ improvement of gait spatiotemporal characteristics (increase of swing time and stride length $(p<0.05)$, together with decrease of stance time and cadence, as well increase of stride length) was noted in the involved leg. In TKA patients 6 months after reoperation due to PFPS the knee joint function in involved leg was significantly improved and the positive changes in gait with comfortable velocity took place as compared before surgery.

Key words: joint replacement, isometric contraction, osteoarthritis, anterior knee pain

\section{INTRODUCTION}

Patellofemoral complications following total knee arthroplasty (TKA) continue to be a significant source of postoperative morbidity and revision surgery. The possible complications include patellofemoral instability, extensor mechanism impairment, soft tissue impingement, prosthetic wear or loosening, and osteonecrosis [18]. Patellofemoral pain syndrome (PFPS) can be defined as anterior knee pain or retropatellar pain in the absence of other specific pathology [6].

Cooney et al [5] retrospectively reviewed 361 patients who had a revision total knee arthroplasty done for an extensor mechanism problem to assess the prevalence, etiology, and risk factors of subsequent reoperation. The prevalence of reoperation was $23 \%$ and 84 patients were reoperated for one or more times. The average time until the first reoperation was 2.4 years. The cumulative risk of a reoperation for any reason was $7 \% 1$ year, $19.6 \% 5$ years, and $35.9 \%$ 10 years after index revision. The authors found that the most common reason for reoperation was a new or recurrent patellofemoral problem, which accounted for $33 \%$ of the first reoperations. By data of Sierra et al [23] the average time from index revision total knee arthroplasty to the first reoperation was 3.5 years (range 1 day19 years) and the cumulative risks of the first reoperation at 5, 10, and 15 years were $16.1 \%, 26 \%$, and $31.4 \%$, respectively.

A retrospective analysis of 2188 total knee arthroplasties performed during six years (2002-2007) in Tartu University Hospital revealed that reoperation was conducted in $2.1 \%$ of cases (47 patients). Reoperation burden was $1.5 \%$ in unconstrained GEMINI SL (LINK), $2.6 \%$ in semiconstrained PFC (De Puy) and $1.3 \%$ in 
constrained rotational hinged knee (LINK). Normal alignment of the $\mathrm{PF}$ joint in the axial X-ray was differentiated in 3 patients $(6.4 \%)$ and other had lateral pressure syndrome - 34 patients $(72.3 \%)$ and patellar subluxation or necrosis - 10 patients $(21.3 \%)$ [11].

The level of ROM limitation and joint contracture localization define the functional limitations of a patient [4, 16]. It has been suggested that an imbalance between the vastus medialis and vastus lateralis muscle forces causes abnormal tracking of patella, resulting in reduced contact areas, increased stresses, and patellofemoral pain [3]. Knee extensor (KE) muscle forces play an important role in determining the medial-lateral force balance, contact force and pressure distribution of the patellofemoral joint $[3,8,13]$. In a review article [2] on kinematic gait characteristics associated with PFPS it was noted that during walking, individuals with PFPS can exhibit impaired ankle joint kinematics and a possible reduction in gait velocity. Previously, it has been demonstrated that persistent gait abnormalities are comparable between patients with different type of knee joint prostheses (posterior-stabilized, cruciate-retaining and cruciate-sacrificing prostheses) [26]. The data regarding the gait biomechanics for TKA patients with PFPS patients is not well documented.

The aim of our study was to investigate the gait and thigh muscle characteristics in TKA patients with PFPS before and six months after surgery. It was hypothesized that gait velocity and other kinematic data can be improved in TKA patients with PFPS 6 months after surgery.

\section{MATERIALS AND METHODS}

\section{Subjects}

Eight patients ( 4 men and 4 women, mean age 66 years, range 56-77; BMI $31.9 \mathrm{~kg} / \mathrm{m}^{2}$, range 26-39) with PFPS following unilateral TKA participated in the study before and six months after surgery (Table 1). Pre-TKA, all patients had primary degenerative knee OA in stage III or IV according to Kellgren-Lawrence Scale and were scheduled for the first TKA. The patients had patellofemoral compartment involvement in $\mathrm{OA}$ in the operated knee joint. The duration of symptoms before TKA was $9.3 \pm 2.5$ years (range $3-20$ years) and reoperation due to PFPS was performed $18.8 \pm 3.5$ months (range 6- 
36 months) later. Patients in the PFPS group were confirmed with the presence of pain and/or tenderness upon palpation of the patella; experienced diffuse anterior knee pain for at least 6-12 months and had increased knee pain when descending stairs and during physical activity. Exclusion criteria were any other joint replacement of lower limbs, other orthopaedic dysfunctions, rheumatoid arthritis, neuromuscular or neurodegenerative diseases, significant cardiac or pulmonal problems. The patients were evaluated twice: preoperatively and six months after reoperation. Before the beginning of the testing session, all subjects completed an informed consent form. The study carried the approval of the Ethics Committee of the University of Tartu.

\section{Measures}

Surgical procedures

For TKA, the condylar endoprostheses GEMINI (W. Link Gmbh \& Co, Germany) with a rotating plateau were used in case of moderate knee varus deformity (up to $10^{\circ}$ ) and stable knee ligaments (in 4 patients). By using this type of prostheses the posterior cruciate ligament was retained. The condylar endoprostheses PFC with posterior joint stabilization (Johnson \& Johnson, USA) were used in case of more severe knee varus deformity (up to $20-25^{\circ}$ ) and instable knee ligaments (in 4 patients). The posterior cruciate ligament was sacrificed in these cases. A fixed polyethylene insert was used. The patella was not resurfaced.

Two kinds of reoperations were performed in TKA patients with PFPS - patella endoprosthesis (resurfacing) or patella resection (reshaping).

\section{Active range of motion}

The knee flexion, extension, and hip abduction and adduction active range of motion (AROM) were measured by standard mechanical goniometer Gollehon Extendable Goniometer (Lafayette Instrument, USA) and standard guidelines [4]. Three measurements were taken for each joint and the best result was accepted for future analysis. 
Isometric maximal voluntary contraction force

Thigh muscle isometric maximal voluntary contraction (IMVC) force in flexion, extension, abduction and adduction was measured by handheld dynamometer (Lafayette Manual Muscle Test System, Lafayette Instrument Company, USA). Hand-held dynamometer is a reliable and valid assessment tool for measuring strength of the hip and knee muscle in older adults [1].

During the IMVC force testing of knee extensor muscle the subject was in seated position on a therapeutic table with knee flexed to aproximately $90^{\circ}$. IMVC testing of knee flexor muscle was performed in prone subject, flexion of the knee between $50^{\circ}$ and $70^{\circ}$ [12]. The subjects were required to exert maximum strength, pushing against the dynamometer for approximately $3 \mathrm{~s}$ as hard as possible with rest period of $1 \mathrm{~min}$. The noninvolved leg was tested first, followed by the involved leg. The best result from three trials was taken as isometric maximal voluntary strength. All assessments were performed by the same physiotherapist.

\section{Gait characteristics}

Gait kinematic and kinetic characteristics were recorded using the ELITE motion analyzer with six infrared cameras with a sampling rate of $100 \mathrm{~Hz}$ and Elite Clinic software (BTS SpA, Italy). Twenty passive reflecting spherical markers with the diameter of $15 \mathrm{~mm}$ were attached to the selected anatomic points of body according to the Davis protocol [7]: three markers on the shoulders, three markers on the pelvis, three markers on each thigh, three markers on each calf and one marker on each foot. The subjects were requested to walk with at their comfortable velocity along a 5.3-m walkway. Three to five trials were recorded and the trial with stable walking cadence (without deor accelerations) was taken for analysis.

The following spatiotemporal parameters of gait were recorded: walking speed, cadence, stride length, stance and swing time, singlelimb and double-limb support time. Kinematic and kinetic characteristics of knee joint flexion and extension - range of motion and corresponding moment of force of knee joint flexion and extension were estimated. Gait characteristics were normalized to gait cycle (cycle duration $=100 \%)$. 
Knee joint pain

Self-estimation of pain and stiffness of knee joint and physical function during recent 48 hours was performed using Western Ontario and McMaster Universities Index of Osteoarthritis (WOMAC) questionnaire [15]. The questionnaire includes 24 questions, five of which estimate pain, two - stiffness and 17 - functional ability. Smaller scores characterize greater impairment of function. Pain subscale (state without pain gives 20 points) and physical activity subscale (state with better physical activity gives 68 points) are included in Table 1.

\section{Statistical analysis}

Data are means and standard errors of means $( \pm \mathrm{SE})$ and range (minmax). Student's paired t-test was used to find differences of data before and after reoperation and unpaired test to compare data of the involved and uninvolved leg. For WOMAC scores statistical analysis was performed using non-parametric tests (normality of scores distribution was tested within each group using the KolmogorovSmirnov test). Comparison of the post- to the preoperative scores was estimated by Wilcoxon test. A level of $\mathrm{p}<0.05$ was selected to indicate statistical significance.

\section{RESULTS}

In the present study TKA patients with PFPS reported to have less pain in knee joint and improved physical function $(p<0.01)$ estimated by WOMAC scores (Table 1). The involved leg's knee flexion AROM was significantly lower as compared to the uninvolved leg pre- and post-surgery (Figure 1A). This characteristic of the involved leg did not change significantly $(\mathrm{p}>0.05)$ post-surgery as compared before it. The patients had greater hip abduction AROM of the involved leg 6 months after surgery as compared before it $(p<0.05)$ (Figure 1B). AROM of knee extension and hip adduction of the involved leg 6 months post-surgery did not differ significantly $(p<0.05)$ from pre-surgery data (data are not shown here). 
Table 1. Anthropometric characteristics and WOMAC scores of TKA patients with PFPS before and 6 months after surgery $(n=8)$

\begin{tabular}{|l|c|c|c|c|c|c|c|c|}
\hline & \multicolumn{4}{|c|}{ Before surgery } & \multicolumn{3}{c|}{ 6 months after surgery } \\
\cline { 2 - 9 } Characteristics & Mean & SE & min & max & mean & SE & min & max \\
\hline Age (year) & 66.3 & 2.3 & 58 & 77 & 66.4 & 2.3 & 58 & 77 \\
\hline Height $(\mathrm{cm})$ & 169.4 & 4.6 & 148.5 & 189.2 & 169.7 & 3.4 & 148.5 & 189.2 \\
\hline Body mass $(\mathrm{kg})$ & 86.3 & 3.8 & 73.4 & 97.9 & 85.7 & 4.0 & 77.2 & 100.4 \\
\hline BMI $\left(\mathrm{kg} / \mathrm{m}^{2}\right)$ & 30.2 & 1.3 & 25.6 & 35.1 & 29.9 & 1.0 & 26.1 & 34.7 \\
\hline $\begin{array}{l}\text { WOMAC-Pain } \\
(0-20)\end{array}$ & 5.4 & 1.2 & 5 & 12 & $12.0 * *$ & 1.7 & 7 & 19 \\
\hline $\begin{array}{l}\text { WOMAC-PF (0- } \\
\text { 68) }\end{array}$ & 18.0 & 3.2 & 7 & 25 & $36.1^{* *}$ & 3.4 & 16 & 45 \\
\hline
\end{tabular}

TKA - total knee arthroplasty; PFPS - patellofemoral pain syndrome; BMI - body mass index, WOMAC - Western Ontario and McMaster Universities Index of Osteoarthritis questionnaire; WOMAC-Pain - pain subscale and WOMAC-PF - physical function subscale (smaller scores characterise greater impairment of function; estimation involved recent 48 hours before testing). Data are presented as mean \pm SE and range (min$\max ) .{ }^{*} \mathrm{p}<0.01$ as compared with data before surgery.

Six months after surgery lower IMVC force $(p<0.05)$ of the involved leg in TKA patients with PFPS was noted as compared to the uninvolved leg (Figure 1C). The patients also demonstrated lower IMVC force $(\mathrm{p}<0.05)$ of the involved leg's hip abductor muscle presurgery as compared to the non-involved leg (Figure 1D). No significant differences were noted in this characteristic 6 months after surgery between the involved and uninvolved leg. 

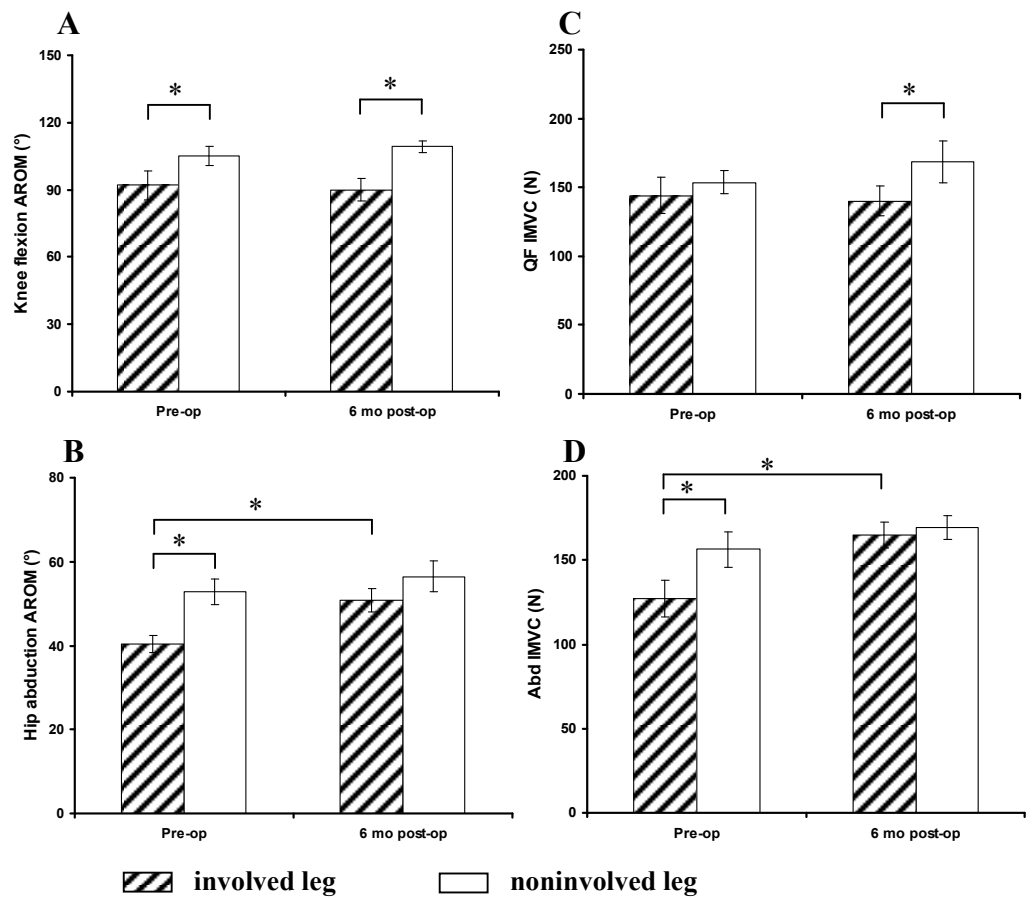

Figure 1. Active range of motion (AROM) and isometric maximal voluntary contraction (IMVC) force of knee joint flexion (A and $\mathbf{C}$, respectively) and hip abduction (B and $\mathbf{D}$, respectively) in total knee arthroplasty patients with patellofemoral pain syndrome before and six months after reoperation (mean and SE, $* \mathrm{p}<0.05$ ).

Six months after surgery a significant increase of swing time $(p<0.01)$ and swing time as per cent of stride $(p<0.05)$ and stride length $(p<0.05)$ of the involved leg were noted in patients as compared to pre-surgery (Table 2). Postoperatively patients demonstrated a decrease $(p<0.05)$ of stance time and cadence as well increase $(p<0.05)$ of stride length as compared to before surgery. Changes in spatiofemoral characteristics of gait are presented in Figure 2. The greatest differences of the above mentioned gait characteristics were noted in the swing time value (21\%) (Figure 2). 
Table 2. Spatiotemporal characteristics of gait of TKA patients with PFPS before and 6 months after surgery (mean and SE, $n=8$ )

\begin{tabular}{|c|c|c|c|c|c|c|}
\hline \multirow[b]{2}{*}{ Characteristics } & \multirow[t]{2}{*}{ Leg } & \multicolumn{2}{|c|}{$\begin{array}{c}\text { Before surgery } \\
1\end{array}$} & \multicolumn{2}{|c|}{$\begin{array}{c}6 \text { months after } \\
\text { surgery } \\
2 \\
\end{array}$} & \multirow[t]{2}{*}{$\underset{(1-2)}{p}$} \\
\hline & & Mean & SE & Mean & SE & \\
\hline \multirow{2}{*}{ Stance time (ms) } & IL & 714.1 & 38.7 & 761.3 & 41.0 & NS \\
\hline & NIL & 727.4 & 44.1 & 746.9 & 34.6 & $\mathrm{NS}$ \\
\hline \multirow{2}{*}{ Swing time (ms) } & IL & $376.5 *$ & 28.9 & 474.4 & 26.8 & 0.008 \\
\hline & NIL & 436.8 & 16.0 & 475.6 & 23.6 & NS \\
\hline \multirow{2}{*}{$\begin{array}{l}\text { Double support } \\
\text { time }(\mathrm{ms})\end{array}$} & IL & 133.5 & 15.9 & 135.0 & 11.2 & NS \\
\hline & NIL & 125.4 & 12.4 & 141.3 & 16.2 & NS \\
\hline \multirow{2}{*}{$\begin{array}{l}\text { Stance time } \\
\text { (\% stride) }\end{array}$} & IL & 66.6 & 1.3 & 61.1 & 0.9 & 0.023 \\
\hline & NIL & 62.0 & 1.3 & 61.5 & 1.3 & $\mathrm{NS}$ \\
\hline \multirow{2}{*}{$\begin{array}{l}\text { Swing time } \\
\text { (\% stride })\end{array}$} & IL & $33.4^{*}$ & 1.3 & 38.9 & 0.9 & 0.023 \\
\hline & NIL & 38.0 & 1.5 & 38.5 & 1.3 & NS \\
\hline \multirow{2}{*}{$\begin{array}{l}\text { Double support } \\
\text { time }(\% \text { stride })\end{array}$} & IL & 12.3 & 0.9 & 10.9 & 0.5 & NS \\
\hline & NIL & 11.6 & 0.9 & 11.3 & 0.8 & NS \\
\hline \multirow{2}{*}{$\begin{array}{l}\text { Stride length } \\
(\mathrm{cm})\end{array}$} & IL & 106.5 & 4.7 & 118.1 & 4.6 & 0.050 \\
\hline & NIL & 108.1 & 7.0 & 113.0 & 4.2 & NS \\
\hline $\begin{array}{l}\text { Stride width } \\
\text { (cm) }\end{array}$ & & 10.4 & 0.7 & 10.2 & 0.6 & NS \\
\hline Velocity $(\mathrm{m} / \mathrm{s})$ & & 0.96 & 0.07 & 0.97 & 0.07 & NS \\
\hline $\begin{array}{l}\text { Cadence } \\
\text { (step/min) }\end{array}$ & & 108.5 & 4.9 & 99.8 & 4.1 & 0.031 \\
\hline
\end{tabular}

TKA - total knee arthroplasty; PFPS - patellofemoral pain syndrome; $\mathrm{IL}$ - involved leg; NIL - noninvolved leg; *p $<0.05$ as compared involved leg with noninvolved leg; NS - not significant difference $(\mathrm{p}<0.05)$ as compared data before and after surgery. 


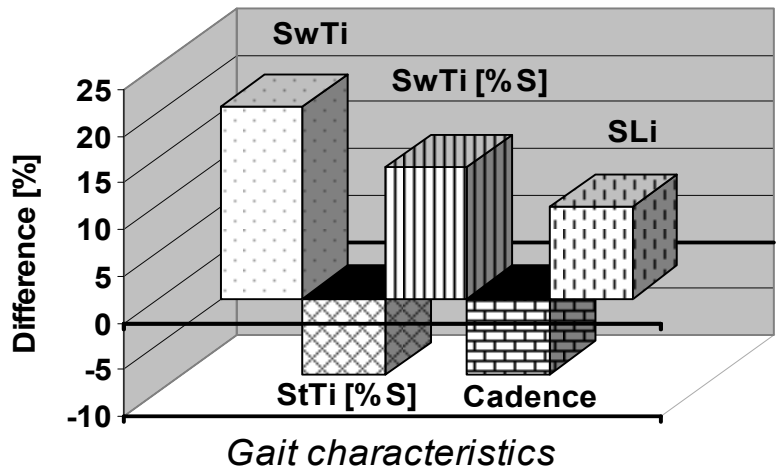

Figure 2. Changes in spatiotemporal characteristics of gait in total knee arthroplasty patients with patellofemoral pain syndrome six months after reoperation $(\mathrm{p}<0.05)$. Data before reoperation was taken as $100 \%$. SwTi swing time in ms $(\mathrm{p}=0.008)$, SwTi $[\% \mathrm{~S}]$, swing time as per cent of stride $(p=0.023), S L i-$ stride length $(p=0.050) ; \operatorname{StTi}[\% \mathrm{~S}]-$ stance time in ms of involved leg $(\mathrm{p}=0.023)$.

No significant differences ( $\mathrm{p}>0.05)$ in other temporal and distance, as well as in kinematic and kinetic characteristics of knee joint as compared pre- and post-surgery were found. The tendency to improve the moment of force of knee joint flexion and extension was noted in patients 6 months after surgery (Figure 3 ). 


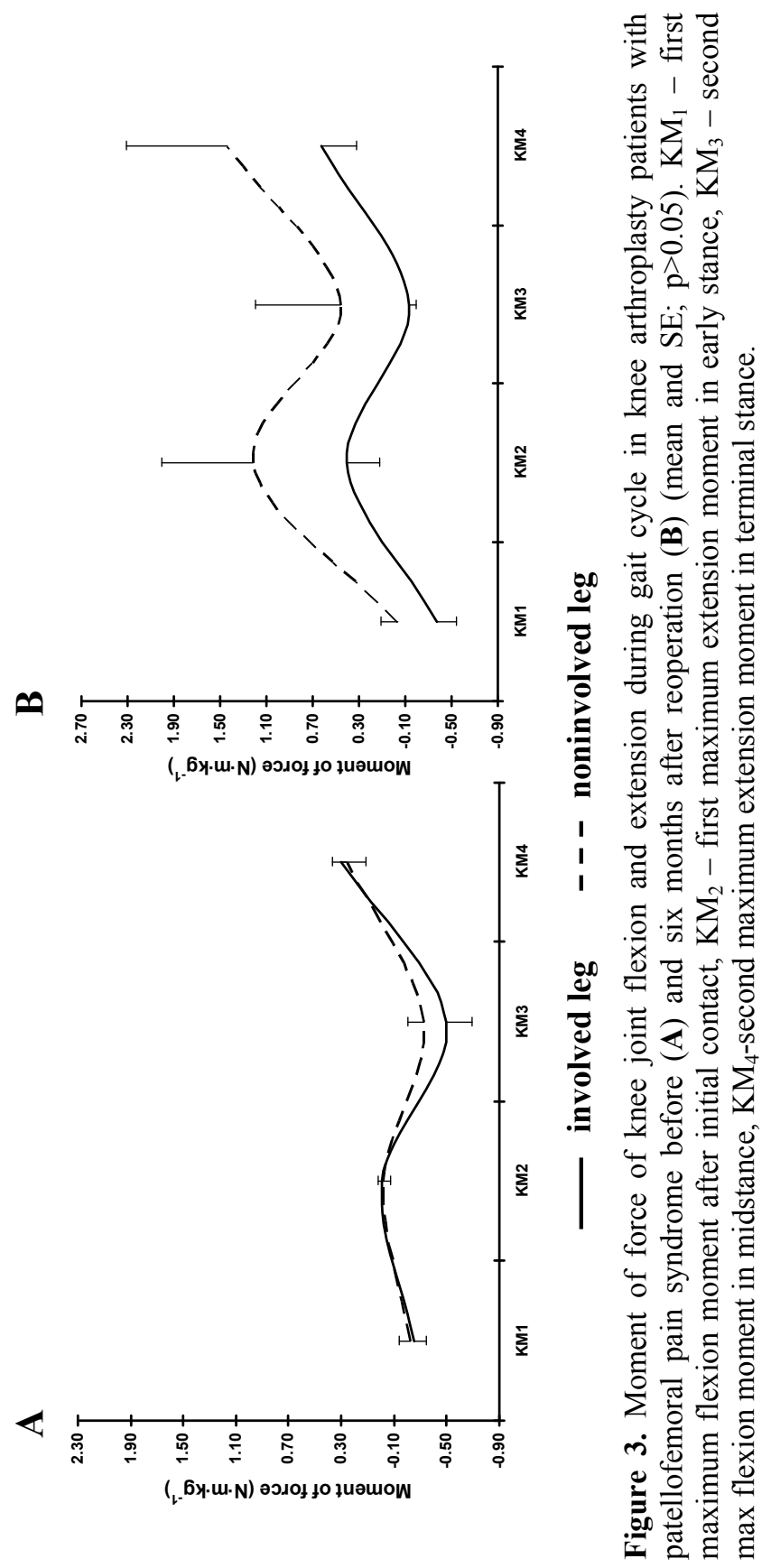




\section{DISCUSSION}

The present study investigated gait spatiofemoral and knee joint kinematic and kinetic characteristics in TKA patients before and 6 months after reoperation due to PFPS. The main finding of the study was that 6 months after surgery take place: (1) significant improvement in selfestimated knee joint function; (2) increase of hip abduction AROM and IMVC force; and (3) improvement of few gait spatiofemoral characteristics (increase of stride length and decrease of gait cadence).

The high mean BMI was noted in patients with post-TKA PFPS. It has been reported that knee joint OA is associated with an increase in body mass and more obese subjects had the greater reduction of the KE strength [24]. In our study, patients demonstrated significant improvement of knee joint function by WOMAC scores after reoperation. In a retrospective study [22] where subjects were TKA patients aged 62-84 years (the post-surgery period was 6-7 years), no statistical significance was noted between men and women concerning differences in anterior knee pain after surgery.

TKA patients with PFPS demonstrated before and 6 months after surgery lower AROM (12\% and 19\%, respectively) and IMVC force ( $8 \%$ and $20 \%$, respectively) of the involved leg for knee flexion as compared to the uninvolved leg. The incidence of flexion deformity following TKA is shown as high as $17 \%$ [25], but no differences were noted in knee extension of stance phase between TKA patients and healthy controls [10]. Previously it has been found that quadriceps muscle strength decrease in patients with orthopaedic problems evokes changes in body balance and gait [16].

In the present study, patients demonstrated significant increase (31\%) of IMVC force of the involved leg's hip abductors postoperatively as compared before surgery. The importance of paying attention to appropriate balancing of the patellofemoral soft tissues was emphasized in studies of patellofemoral pain after TKA [20] and of the effect of soft tissues on patellar tracking [21].

Significant improvement of gait spatiofemoral characteristics was noted in the present study 6 months after surgery - greater increase (prolongation was found in swing time $(21 \%)$, this was accompanied with shortening of stance time distribution in gait cycle (Figure 2). As a result of improvement of hip abductor strength, the gait stride was $8 \%$ longer and cadence was $9 \%$ less post-surgery as compared before it. The study of McClelland et al [14] demonstrated the ability of TKA 
patients to increase walking speed by adjusting spatiotemporal parameters. From comfortable to fast walking the velocity in the TKA group was increased similarly to healthy controls - as a result of an increase in both stride length and cadence. 6 months after surgery no significant improvement in gait velocity was noted. Patients in the present study had lower gait velocity $(0.96$ pre- and $0.97 \mathrm{~m} / \mathrm{s}$ postsurgery) and cadence as compared to the data of Besier et al [3] who found that PFPS subjects walked and ran with similar speed, stride length, and cadence to the control groups of males and females.

TKA patients with PFPS had no significant improvement in kinematic and kinetic characteristics of knee joint after surgery as compared pre-operatively. The tendency to improve the moment of force of knee joint flexion and extension was noted - in first maximum extension in early stance (mean increase of $85 \%$ ) and in second maximum extension moment in terminal stance (increase of 23\%). The study of Ouelett and Moffet [17] demonstrated that TKA patients walked with a more flexed hip in stance phase and a less flexed knee and plantarflexed ankle during early stance and had a significant decrease in extensor and flexor moments at the hip and knee throughout the gait cycle. It has been found that at the knee joint, altered tibiofemoral rotation and an increase of knee adduction during the stance phase of gait result in lateral patellar tracking and increase lateral patellofemoral joint compression [19]. It has been found that compared to healthy controls, the PFPS group had greater cocontraction of quadriceps and hamstrings during walking, even though the net knee moment was similar between groups [3].

The limitation of the present study is a small number of patients who were evaluated.

In conclusion, the study demonstrated that in TKA patients 6 months after reoperation due to PFPS the knee joint function was significantly improved - hip abduction active range of motion and isometric force were increased. At the same time the positive changes in gait with comfortable velocity were found: increase of the stride length and decrease of cadence as well as normalisation of gait cycle phases took place. 


\section{ACKNOWLEDGEMENTS}

This study was supported by the Estonian Ministry of Education and Research project No SF0180030s07 and Estonian Science Foundation project No 7939.

\section{REFERENCES}

1. Arnold C. M., Warkentin K. D., Chilibeck P. D., Magnus C.R. (2010) The reliability and validity of handheld dynamometry for the measurement of lower-extremity muscle strength in older adults. J. Strength Cond. Res. 24: 815-824

2. Barton C. J., Levinger P., Menz H. B., Webster K. E. (2009) Kinematic gait characteristics associated with patellofemoral pain syndrome: A systematic review. Gait Posture. 30: 405-416

3. Besier T. F., Fredericson M., Gold G. E., Beaupré G. S., Delp S. L. (2009) Knee muscle forces during walking and running in patellofemoral pain patients and pain-free controls. J. Biomech. 42: 898905

4. Clarcson H. M. (2005) Joint motion and function assessment. Lippincott, Williams \&Wilkins, Philadelphia

5. Cooney W. P. 4th, Sierra R. J., Trousdale R. T., Pagnano M. W. (2005) Revision total knees done for extensor problems frequently require reoperation. Clin. Orthop. Relat. Res. 440: 117-121

6. Crossley K., Cowan S., Green S., McConnel J. (2001) A systematic review of physical interventions for patellofemoral pain syndrome. Clin. J. Sport Med. 11: 103-110

7. Davis R. B., Ounpuu S., Tyburski D. S., Gage J. R. (1991) A gait analysis collection and reduction technique. Hum. Mov. Sci. 10: 575587

8. Elias J. J., Bratton D. R., Weinstein D. M., Cosgarea A. J. (2006) Comparing two estimations of the quadriceps force distribution for use during patellofemoral simulation. J. Biomech. 39: 865-872

9. Fergusson D., Hutton B., Drodge A. (2007) The epidemiology of major joint contractures: a systematic review of the literature. Clin. Orthop. Relat. Res. 456: 22-29

10. Fuchs S., Floren M., Skwara A., Tibesku C. D. (2002) Quantitative gait analysis in unconstrained total knee arthroplasty patients. Int. J. Rehabil. Res. 25: 65-70

11. Haviko T., Märtson A., Parv M. (2009) Jacques Duparc Award. $10^{\text {th }}$ EFORT Congress, Vienna, Austria, 3-6 June 2009, P708 
12. Kendall F. P., McCreary E. K., Provance P. G., Rodgers M. M., Romani W. A. (2005) Muscles testing and function with posture and pain. $5^{\text {th }}$ edn. Lippincott Williams \& Wilkins, Baltimore

13. Lee T. Q., Sandusky M. D., Adeli A., McMahon P. J. (2002) Effects of simulated vastus medialis strength variation on patellofemoral joint biomechanics in human cadaver knees. J. Rehabil. Res. Dev. 39: 429-438

14. McClelland J. A., Webster K. E., Feller J. A., Menz H. B. (2011) Knee kinematics during walking at different speeds in people who have undergone total knee replacement. Knee. 18: 151-155

15. McConnel S., Kolopack P., Davis A. M. (2001) The Western Ontario and McMaster Universities Osteoarthritis Index (WOMAC): a review of its utility and measurement properties. Arthritis Rheum. 45: 453461

16. Mollinger L. A., Steffen T, M. (1993) Knee flexion contractures in institutionalized elderly: prevalence, severity, stability, and related variables. Phys. Ther. 73: 437-446

17. Ouellet D., Moffet H. (2002) Locomotor deficits before and two months after knee arthroplasty. Arthritis Rheum. 47: 484-493

18. Parker D. A., Dunbar M. J., Rorabeck C. H. (2003) Extensor mechanism failure associated with total knee arthroplasty: prevention and management. J. Am. Acad. Orthop. Surg. 11: 238-247

19. Powers C. M. (2003) The influence of altered lower-extremity kinematics on patellofemoral joint dysfunction: a theoretical perspective. J. Orthop. Sports Phys. Ther. 33: 639-646

20. Scuderi G. R., Insall J. N., Scott N. W. (1994) Patellofemoral pain after total knee arthroplasty. J. Am. Acad. Orthop. Surg. 2: 239-246

21. Senavongse W., Bull A. M. J., Taylor A. R., Amis A. A. (1998) Muscle imbalance alone does affect patellar tracking: an in-vitro investigation. J. Bone. Joint Surg. Br. 80B (Suppl 3): 258

22. Sensi L., Buzzi R., Giron F., De Luca L., Aglietti P. (2011) Patellofemoral function after total knee arthroplasty: sex-related differences. J. Arthroplasty. 26: 1475-1480

23. Sierra R. J., Cooney W. P. 4th, Pagnano M. W., Trousdale R. T., Rand J. A. (2004) Reoperations after 3200 revisions TKAs: rates, etiology, and lessons learned. Clin. Orthop. Relat. Res. 425: 200-206

24. Slemenda C., Heilman D. K., Brandt K. D., Ktz B. P., Mazzuca S. A., Braunstein E. M., Byrd D. (1998) Reduced quadriceps strength relative to body weight: a risk factor for knee osteoarthritis in women. Arthritis Rheum. 41: 1951-1958 
25. Tew M., Forster I. W., Wallace W. A. (1989) Effect of total knee arthroplasty on maximal flexion. Clin. Orthop. Rel. Res. 247: 168174

26. Wilson S. A., McCann P. D., Gotlin R. S., Ramakrishnan H. K., Wootten M. E., Insall J. N. (1996) Comprehensive gait analysis in posterior-stabilized knee arthroplasty. J. Arthroplasty. 11: 359-367

\section{Correspondence to:}

Helena Gapeyeva

Institute of Exercise Biology and Physiotherapy

University of Tartu

Jakobi 5, 51014 Tartu

Estonia

E-mail: helena.gapeyeva@ut.ee 